\title{
Comment on: 'Association of Chlamydia trachomatis ompA genovar with trachoma phenotypes'
}

\author{
Hugh Taylor ${ }^{1}$
}

Received: 30 April 2018 / Accepted: 1 June 2018 / Published online: 2 November 2018

(c) The Royal College of Ophthalmologists 2018

I was interested to read the paper by Chin et al. [1] that looked at the association of Chlamydia trachomatis ompA genovars and trachoma phenotypes. In their study in Ethiopia, they found that the $93 \mathrm{~B}$ genotypes were somewhat more likely to be associated with more severe disease than the 258A genotypes that they identified. Although their report is brief, they may be interested in studies done in a non-human primate mode that did show that there is not only some variation between different serovars in the clinical response they induce, but also some variation within different strains of serovar [2].

Hugh Taylor

h.taylor@unimelb.edu.au

1 University of Melbourne, Melbourne, Australia
At the time this previous work was done, it was not possible to genotype isolates and so the genetic variation is unknown, although the clinical variation is also quite marked.

\section{Compliance with ethical standards}

Conflict of interest The authors declare that they have no conflict of interest.

\section{References}

1. Chin SA, Alemayehu W, Melese M, et al. Association of Chlamydia trachomatis ompA genovar with trachoma phenotypes. Eye. 2018; 32:1411-1420.

2. Taylor HR. Development of immunity to ocular chlamydial infection. Am J Trop Med Hyg. 1990;42:358-364.

\section{Are patients with ectopia lentis known to cardiology services?}

\author{
P. Maghsoudlou (iD ${ }^{1} \cdot$ T. Khanam ${ }^{1} \cdot$ P. J. Banerjee ${ }^{1,2} \cdot$ A. Chandra ${ }^{1,3}$
}

Received: 15 September 2018 / Accepted: 25 September 2018 / Published online: 5 November 2018

(c) The Royal College of Ophthalmologists 2018

P. Maghsoudlou

p.maghsoudlou@ucl.ac.uk

1 UCL Institute of Ophthalmology, University College London, London EC1V 9EL, UK

2 Department of Ophthalmology, Frimley Park Hospital, Frimley GU16 7UJ, UK

3 Department of Ophthalmology, Southend University Hospital, Southend SSO ORY, UK
Ectopia lentis (EL) is the malposition of the natural lens caused by a defect in the ciliary zonules [1]. The most common cause for EL is Marfan syndrome (MFS) [2]. EL occurs in $~ 70 \%$ of patients with MFS $[3,4]$. Up to $100 \%$ of patients with MFS have an abnormal cardiovascular system predisposing to increased mortality and morbidity [5]. Echocardiography is the mainstay of cardiological assessment for patients with MFS [6]. 
Ophthalmologists are well aware of the ocular risks of EL and routinely survey for these. However, ensuring cardiovascular assessments may be less-regularly documented. Herein we audit documentation of whether patients with EL are known to cardiologists, geneticists or physicians for their systemic cardiovascular risks.

Eligible patients were identified from the database of a tertiary ophthalmic centre: Moorfields Eye Hospital, London. A database search between 1 January 1959 and 31 December 2011 was performed. The inclusion criteria were all referrals for EL or for syndromes associated with EL. Patients were excluded if the EL diagnosis was not confirmed, or if patients were subsequently seen elsewhere.

The standard of the audit was that all patients should have documentation that the patient is under cardiovascular review.

In all, 191 patients fulfilled the inclusion criteria. Ninetyseven patients $(51 \%)$ had documentation that they were under cardiovascular review. Of these, 38 (20\%) were documented to be known as having cardiovascular issues, while 59 patients (31\%) did not. A total of 94 patients (49\%) had no documentation regarding cardiovascular problems.

Our findings describe the lack of documentation ensuring cardiovascular review for patients with EL. Ophthalmologists are well aware that the most common cause of EL is MFS and associated cardiovascular risks. It is important for patient safety that these patients are known to cardiology or medical services. Ophthalmologists first see $\sim 40 \%$ of patients with MFS [7]. It is critical therefore to ensure that patients with EL are under the care of appropriate physicians. There are currently no guidelines on referral pathways for patients who present to ophthalmologists with EL, suggesting the need to review investigation and management of this cohort.

\section{Compliance with ethical standards}

Conflict of interest The authors declare that they have no conflict of interest.

\section{References}

1. Stone JH. Ectopia lentis, cardiology, and the sign of the tremulous iris. Am Heart J. 1966;72:466-8.

2. Chandra A, Charteris D. Molecular pathogenesis and management strategies of ectopia lentis. Eye 2014;28:162-8.

3. Marsalese DL, Moodie DS, Vacante M, Lytle BW, Gill CC, Sterba $\mathrm{R}$, et al. Marfan's syndrome: natural history and long-term followup of cardiovascular involvement. J Am Coll Cardiol. 1989;14: 422-8-31.

4. Rubin S, Nelson L. Ocular manifestations of autosomal dominant systemic conditions. In: Tasman W, Jaegar E, editors. Duane's clinical ophthalmology. 2nd ed. Lippincott Williams and Wilkins; Philadelphia 2006.

5. Graham Stuart A, Williams A. Marfan's syndrome and the heart. Arch Dis Child. 2007;92:351-6.

6. Child AH. Diagnosis and management (Summary). In: Diagnosis and management of Marfan Syndrome. (ed.) Child, Anne H. London: Springer; 2016. p. 3-11.

7. Strider D, Moore T, Guarini J, Fallin B, Ivey J, Kron I. Marfan's syndrome: a family affair. J Vasc Nurs. 1996;14:91-98.

\title{
Periocular basal cell carcinoma in under 40's - Is more aggressive treatment warranted?
}

\author{
Edward Saxby $^{1} \cdot$ Mark Wright $^{1} \cdot$ Rhys Davies $^{1}$
}

Received: 15 November 2018 / Accepted: 15 November 2018 / Published online: 5 December 2018

(c) The Royal College of Ophthalmologists 2018

Edward Saxby

e_saxby@hotmail.com

1 Princess Alexandra Eye Pavilion, Chalmers Street, Edinburgh EH3 9HA NHS Lothian, UK
Basal cell carcinoma (BCC) is the most common form of skin cancer. It is slow growing and locally invasive. Risk factors include ultraviolet light exposure, increasing age, fair skin, immunosuppresion and familial conditions e.g. Gorlins Syndrome [1]. BCCs are commonly diagnosed in elderly populations but are still rarely diagnosed in youth [2]. We investigated the incidence, treatment and outcome 